\title{
Criminologie
}

\section{Dangerosité et justice : la peur du criminel ou la peur de la criminologie?}

\section{Pierre Lagier et André Normandeau}

Volume 15, numéro 2, 1982

Criminels et psychiatrie

URI : https://id.erudit.org/iderudit/017163ar

DOI : https://doi.org/10.7202/017163ar

Aller au sommaire du numéro

Éditeur(s)

Les Presses de l'Université de Montréal

ISSN

0316-0041 (imprimé)

1492-1367 (numérique)

Découvrir la revue

Citer ce compte rendu

Lagier, P. \& Normandeau, A. (1982). Compte rendu de [Dangerosité et justice :

la peur du criminel ou la peur de la criminologie ?] Criminologie, 15(2),

105-114. https://doi.org/10.7202/017163ar d'utilisation que vous pouvez consulter en ligne.

https://apropos.erudit.org/fr/usagers/politique-dutilisation/ 
DANGEROSITÉ ET JUSTICE : LA PEUR DU CRIMINEL OU LA PEUR DE LA CRIMINOLOGIE? *

Pierre Lagier

André Normandeau

La notion de dangerosité a-t-elle encore un sens pour le criminologue-praticien qui, au Québec ou ailleurs, est confronté quotidiennement à un "client " qui "fait peur au monde" ? Le moins que l'on puisse dire, à la lumière de la littérature des dernières années sur le sujet, c'est que ce «sens » est désorienté, contesté et remis radicalement en question, non seulement par les universitaires et les théoriciens mais également par les praticiens eux-mêmes. Une recherche québécoise en cours auprès des praticiens (Dozois, Lalonde et Poupart) nous livrera d'ici peu des précisions sur les perceptions de ces derniers. Nous laisserons de côté ce volet. En contrepartie, compte tenu de la parution récente d'un livre important sur le concept de dangerosité, qui est en fait un collectif rédigé sous la direction d'un criminologue d'orientation clinique, Christian Debuyst (1981), nous aimerions signaler au lecteur de la revue Criminologie quelques-unes des interrogations critiques les plus intéressantes.

Ce livre, intitulé : Dangerosité et justice pénale : ambiguitté d'une pratique, est le résultat d'un colloque sur le thème de la dangerosité qui a marqué le cinquantième anniversaire de l'École de criminologie de Louvain, en Belgique. Le "maître de cérémonie ", Christian Debuyst, n'en était pas à ses premières interrogations sur le sujet. En effet, il en avait déjà largement débattu avec d'autres criminologues dès les années 50 lors des congrès et des cours internationaux de l'époque. Le débat à cette époque, toutefois, était orienté vers le problème limité d'une définition clinique et criminologique adéquate de la dangerosité. Récemment, Debuyst en est arrivé à critiquer sévèrement le concept même de personnalité criminelle et de personnalité dangereuse $(1975,1977,1978,1981,1982)$. En fait, les interrogations ont débouché finalement sur des considérations socio-politiques, idéologiques et ethniques, réflétées par les actualités bibliographiques (Montandon, 1979) et un débat spécifi-

* Dangerosité et Justice pénale. Ambiguitté d'une pratique. Actes du Colloque du $50^{e}$ anniversaire de l'École de criminologie de l'Université de Louvain. Par Christian Debuyst. Genève : Masson, Médecins et Hygiène, 1981. 350 p. (Collection Déviance et Société). 
que (Shah et al., 1981) au sein du collectif ou inter-laboratoire de réflexion et de recherche : Déviance et société. Ce collectif francophone d'origine européenne, auquel se sont associés certains québécois, a été fondé en 1977. Il est responsable, depuis ce temps, de l'édition d'une revue trimestrielle et d'une collection de livres qui portent l'ètiquette du collectif, c'est-à-dire Déviance et société. Le livre sous la direction de Debuyst (1981) qui nous sert de toile de fond pour cette note de lecture en est précisément l'un des produits.

\section{AMBIGUITTÉ D'UNE CRIMINOLOGIE}

Dès l'introduction de l'ouvrage, en annonçant son plan et en rappelant le thème du colloque - la dangerosité a-t-elle encore un sens ? - C. Debuyst annonce une interrogation à partir de deux types de discours. Car, dit-il, certains comme le discours criminologique "prétendent se référer à des données fournies par l'observation objective " alors que "d'autres se situent dans l'ordre de l'élaboration de la règle ou de l'intervention sociale entendue dans un sens général » (p. 4).

Malheureusement, disons-le tout de go, la part faite au "discours criminologique " aura été plutôt mince dans ce colloque, même si une première partie des actes (l'analyse critique de la notion de dangerosité) lui est en principe consacrée par l'étude de la personne dangereuse, de la situation dangereuse et de la représentation de l'homme dangereux. À l'exception du rapport à caractère encyclopédique de P. Lievens sur " l'apport de la psychiatrie à l'utilisation du concept de personnalité dangereuse", c'est en vain en effet que l'on cherchera les données issues de «l'observation objective ». Certes les différents exposés de cette première partie ne manquent ni d'intérêt ni de séduction.

a) À propos du concept de personnalité dangereuse tout d'abord, et avec toutes les nuances souhaitables, $C$. Debuyst critique la criminologie clinique basée sur le concept de personnalité. Les caractéristiques spécifiquement reconnues aux délinquants persistants, dit-il, proviennent d'un point de vue "axéé sur l'idée que le comportement délinquant est l'expression de caractéristiques négatives de la personnalité " (p. 21). Et ce point de vue, poursuit-il, "restreint considérablement les éléments qui nous paraissent être en cause dans une situation conflictuelle susceptible de donner lieu à délinquance " (p. 22). D'où une nécessité de "modifier les perspectives et d'introduire le concept de situation ou de position occu- 
pée par le sujet " (p. 21). Par rapport au groupe ou par rapport à autrui cette position déterminera le sens du comportement. Dans la même section thématique, l'analyse de J. François s'en veut d'ailleurs une sorte de dèmonstration, lorsqu'il explique comment joue le concept de dangerosité à trois moments (trois situations donc) de la vie carcérale : l'entrée du détenu en prison, la prise de décision sur sa libération conditionnelle et sa relation singulière avec le clinicien. Mais ni chez cet auteur, ni chez Debuyst la démonstration n'est totalement convainquante.

Malgré ou peut-être à cause d'un triple recours à De Greeff quant aux instincts de défense et de sympathie, à l'interactionnisme relativement aux phénomènes cognitifs et l'École de Lyon pour son concept de lien sociopathique, la démonstration de Debuyst n'explique pas pourquoi la situation primerait sur la personnalité dans la détermination du sens du comportement. Ou bien en effet on s'en tient à un behaviorisme strict et le comportement n'est que la réponse ad hoc à un stimulus situationnel, ou bien force est d'accepter que la situation aussitôt perçue par le sujet - en l'occurrence ici le délinquant - imprègne sa personnalité tant en ce qui concerne ses mécanismes cognitifs que dans ses instances inconscierfies, au point de contribuer à sa structuration, surtout lorsqu'elle se répète. On pourra même dire que la personnalité, dans une certaine mesure, favorise l'éclosion de situations dont l'orientation behaviorale est précisément toujours la même.

Ả cet égard l'exemple "interactionniste ", choisi par Debuyst, de l'automobiliste en fâcheuse posture, mérite une autre perspective malheureusement délaissée pour la connaissance de la criminalité routière : n'y aurait-il pas une tendance de certaines personnalités à créer des situations (de circulation) conflictuelles, et véritablement «dangereuses"? On peut se demander, par exemple, en quoi la compétence sociale, justement au sens donné par J.P. de Waele (1975), ne constituerait pas une caractéristique de personnalité, - peut-être la plus importante du point de vue criminologique.

b) De même les exemples " cliniques 》 rapportés par l'équipe de Lyon dans la section du recueil consacrée à la représentation de l'homme dangereux pourraient fort bien être analysés dans le sens que nous indiquons.

En effet, Buffard et al, écrivent :

La situation dangereuse implique une mise en place de diffé- 
rents protagonistes dans un espace et un temps qui leur sont communs. Il y aurait donc des moments et des lieux où le danger serait plus menaçant (p. 177).

Certes! Mais pourquoi n'y aurait-il pas aussi des protagonistes plus menaçants? D'un point de vue clinique comme d'un point de vue psycho-sociologique, on sait fort bien qu'une même situation n'est pas vécue de la même façon par les différents acteurs. Pourquoi systématiquement oublier ici les lumineuses distinctions de Kinberg (1959) sur la diversité « des situations dangereuses "?

Une situation est rarement dangereuse en soi, les cliniciens précisément le savent bien, eux qui ont toujours à tenir compte de l'interaction : interaction entre thérapeute et client, entre criminel et victime, "entre homme qui se croit en danger et celui qu'il imagine être dangereux " (p. 188), etc.

À cet égard le petit exposé de I. Cassiers, malgré son tranchant lacanien, est très rafraîchissant. L'homme dangereux, dit-il,

est celui qui menace le refoulement. Mais non pas parce que celui-ci est fragile et, une fois détruit, laisserait libre cours à des pulsions dangereuses pour l'Ordre. Mais parce que le refoulement est la tension nécessaire à chacun pour maintenir l'écart entre ses représentations conscientes et imaginaires, écart dans lequel et par lequel il vérifie son existence (p. 157).

Voilà qui fait comprendre pourquoi malgré sa très faible incidence statistique l'homicide demeure le prince des crimes et le meurtrier celui des criminels. Pourtant les criminologues savent bien que là n'est pas la vraie délinquance et que la plupart des meurtriers sont strictement du ressort psychopathologique.

Aussi doit-on être d'accord avec $\mathrm{Cl}$. Faugeron, en dépit de son analyse un peu trop classiquement marxiste, lorsqu'elle relie la notion de dangerosité au champ idéologique dans lequel elle est utilisée. Le déviant renvoie toujours sa propre image au définisseur de la déviance. Des études cherchant à préciser comment et pourquoi s'élaborent des images différentielles de la déviance et de la dangerosité sont très certainement utiles à la criminologie générale. Mais il ne s'agit pas ici d'un miroir aux alouettes. Peut-être conviendrait-il de se rappeler les grandes règles méthodologiques de la criminologie (cf. Pinatel, 1970, p. 69-77) et ne pas tout confondre trop rapidement : déviance et délinquance, vrais et pseudo-délinquants, criminalité et dangerosité.

c) C'est d'ailleurs le grand mérite de G. Houchon, dans la section consacrée à la situation dangereuse, de remettre un peu les 
choses en place et en perspective. Nous ne venons pas de découvrir la lune, contrairement à ce que semble annoncer J. Goemaere, voyant dans l'approche sociologique « une conception renouvelée de la clinique criminologique" (p. 110). On pourrait d'abord ergoter avec cet auteur sur la pertinence d'assimiler à la sociologie ce qui est depuis longtemps reconnu comme champ de la psychologie sociale. Là n'est pas l'essentiel. Le plus grave en effet nous paraît être dans l'oubli des sources criminologiques. L'étude sociologique du cas individuel, par exemple, ne date pas d'hier (cf. Van Bemmelin, 1950; Mannheim, 1953). Elle est magnifiquement opérationnalisée dans la méthode de J.P. De Waele (1975). Et les travaux mêmes de l'équipe de Louvain confirme sa nécessité. Mais enfin, celle-ci a été reconnue depuis fort longtemps; elle est au coeur même de la multidisciplinarité qui fonde la criminologie, tant générale que clinique. Aussi faudrait-il arrêter de tirer la couverture à soi et de nier à la criminologie la possibilité d'être elle-même, c'est-à-dire interdisciplinaire.

Certes la règle de l'homogénéité des niveaux d'interprétation pose problème, d'autant plus que le champ criminologique se complexifie aujourd'hui. Pourquoi en repousser la solution aux calendes ou dans l'approche macro-sociologique? Houchon semble préconiser son abandon lorsqu'il s'agit d'aborder le micro-social. Il n'indique pas clairement les conditions de son maintien. Il est pourtant urgent de réfléchir à cette question tant est grand le risque de ne plus savoir de quoi on parle, risque dont est signe avant-coureur la proposition de Goemaere de ni plus ni moins donner la réaction sociale comme objet à la criminologie.

Finalement, dans l'ensemble des exposés de cette première partie, deux éléments sont remarquables. Il y a d'une part - effet de la mode ? - un étrange réductionnisme du concept de personnalité qui est peu à peu vidé de tout contenu pour ne plus devenir qu'une vague notion sans la portée opérationnelle qui lui avait été jadis reconnue (cf. Pinatel, 1970, p. 63-65). D'autre part, il y a cette effarante constatation qu'aujourd'hui plusieurs universitaires de renom peuvent disserter longuement sans jamais définir l'objet de leur discours : nulle part en effet la notion de dangerosité n'a été précisée!

Certes, on peut dire avec $\mathrm{Cl}$. Faugeron qu'elle «s'évalue mieux par ses effets sociaux, son usage social que par son contenu positif " (p. 161). Mais enfin ou bien la notion a du sens ou elle n'en a pas. Quant à nous, ce recueil nous a convaincu qu'en criminologie, 
elle n'en a pas. En clinique criminologique on peut d'ailleurs douter qu'elle en ait jamais eu. S'il s'agissait d'évaluer la capacité criminelle spécifique, la criminologie clinique avait déjà le concept de témibilité proposé par Garofalo (cf. Pinatel, 1970, p. 500-501 et 524-527). Il n'apparaît guère utile d'en changer. Le discours criminologique serait-il un autre réductionnisme - assimilé au discours de "l'opinion publique " et dès lors traité en tant que tel c'est-à-dire comme un discours d'opinion.

II. ANALYSE DE LA NOTION DE DANGEROSITÉ DANS LA PROBLEMATIQUE CRIMINELLE

Le second volet de ce livre sur la« dangerosité et justice pénale " porte sur la politique criminelle et la loi. Cette partie relève essentiellement d'une perspective critique, et ouvre la voie, selon l'expression de Christian Debuyst, vers ce qu'on a appelé, avec sans doute beaucoup de naïveté, de nouvelles perspectives pour un compromis social.

Certaines réflexions critiques générales et radicales ouvrent le débat à ce chapitre. Luke Hulsman propose ainsi le paradigme abolitionniste du système de justice pénale, comme le fait d'ailleurs depuis quelques années Nils Christie. Dans ce modèle, le problème social fondamental ne serait plus en aucune manière le « crime" mais le "système de justice pénale " lui-même. La disparition de la notion de crime n'impliquerait pas la disparition de notions telles que "bien et mal", "légal et illégal", "licite et illicite» ou "préjudice".

En fait, le concept-clé serait celui de situations problématiques. La réaction à ces situations ferait appel plus souvent au système de droit civil et administratif plutôt qu'au droit criminel. La conciliation entre les "victimes" et les "criminels » serait développée sur une large échelle par une gestion des conflits qui éviterait que le conflit soit volé à la victime. Ce modèle implique évidemment que le degré de tolérance d'une société soit relativement élevé afin que les styles de contrôle social autres que le contrôle pénal soient valorisés, à savoir : éducatif et travail social, thérapeutique et travail social, compensatoire, et conciliateur. Hulsman fait donc un appel idéologique à un changement socio-culturel important qui permettrait que la "gravité " ou la "dangerosité " d'un événement soit "dédramatisée", "neutralisée" ou, à tout le moins, 
"médiatisée " par un contrôle social de type réparateur plutôt que de type punitif.

Ces «belles pensées » à la Hulsman et Christie apparaissent pour l'instant de l'ordre de l'utopie, compte tenu que le corridor de la tolérance ne semble pas avoir été élargi de façon significative depuis quelques années. Mais le changement social exige toujours un peu d'utopie. Comme le veut le dicton syndicaliste : il faut demander plus pour obtenir le minimum exigé ! Le paradigme abolitionniste est un excellent outil pédagogique pour baliser le changement vers des alternatives réelles et réalistes et pour fouetter l'imagination créatrice à la recherche d'innovations en matière de justice.

Au-delà de cette critique radicale, d'autres contributions méritent notre attention. L'article de Pierre Landreville et Michael Petrunik est de celles-là car leurs interrogations s'inscrivent dans une réalité que nous connaissons bien, celle des législations nord-américaines. Les auteurs soulignent que, malgré les critiques, les législations spéciales concernant les délinquants dits dangereux continuent à proliférer et semblent même connaître un nouvel essor. En particulier, malgré les nombreuses remises en question du rôle des experts, de leur capacité de prédire avec succès le comportement violent et les infractions sexuelles graves, ainsi que l'inefficacité des mesures de détention indéterminée, la notion de dangerosité est devenue une notion passe-partout dans le domaine du droit pénal et de la santé mentale.

Le regain de popularité de cette notion est associé à une tendance à la "bifurcation" dans le domaine des politiques de contrôle social : une "ligne dure " plus sévère pour la délinquance grave de violence et des mesures plus douces pour la délinquance contre les biens. La loi canadienne "paix et sécurité " de 1977, qui proposait une définition du délinquant dangereux encore plus large et plus vague que les précédentes, en est une illustration que les auteurs critiquent sévèrement. Mais pourquoi le concept de dangerosité est-il toujours aussi important dans les législations et les politiques ? Il est probable que l'origine et la persistance des mesures spéciales de contrôle pour les dits dangereux puissent être en partie expliquées par ce que Merton nomme "la perception sociale des problèmes sociaux ». Un seul crime de violence nous préoccupera plus que quelques centaines ou quelques milliers de morts accidentelles sur les routes ou dans les accidents de travail, morts que nous percevons comme normales et routinières. Les crimes de violence soulè- 
vent l'indignation morale, facilitant la tâche des " entrepreneurs moraux " (Becker) dans leur campagne pour faire adopter de nouvelles législations.

Il faut ajouter aussi que ces lois ont des fonctions idéologiques importantes. Comme Landreville et Petrunik le soulignent, les politiciens sont nécessairement sensibles aux préoccupations ou du moins aux pressions des électeurs. Aussi, même lorsqu'un "problème " ne peut être résolu adéquatement, il est important que le gouvernement s'intéresse à la question, propose des solutions et laisse du moins croire qu'il les met en application. Aussi, ces solutions peuvent être "symboliques " plutôt qu'instrumentales (Gusfield). Souvent, il importe peu que la nature et l'étendue du "problème " soient mal évaluées et que les solutions apportées masquent ou déplacent le problème pourvu que le public soit apaisé. Souvent, ceux qui proposent ces solutions veulent croire ou arrivent à croire à ce qui est en réalité des pseudo-solutions. De plus, on peut constater que ces législations attirent l'attention sur le "danger" ou la violence individuelle plutôt que la violence collective ou structurelle. Ce sont les actes des individus, plutôt que les conditions sociales ou des pratiques qui menacent encore plus sérieusement les biens que l'on dit vouloir protéger (la santé, la sécurité, la vie) qui sont perçus et définis comme dangereux.

Les situations ou les conditions de travail mettant en danger la santé des travailleurs, l'ivresse au volant ou la pollution industrielle ne sont pas définies comme dangereuses au point où il faille adopter des mesures spéciales très sévères pour "protéger le public ». En mettant l'accent sur un certain type de violence ou de dangerosité, on détourne l'attention de certains autres problèmes sociaux et politiques. On ignore les questions d'injustice, d'inégalité, les situations répressives qui ont pu engendrer la frustration et l'agression. Le système détourne l'attention des explications sociales, économiques, culturelles et politiques de ce type de comportement. En guise de conclusion, les auteurs s'interrogent ainsi sur les fonctions politiques de la notion de dangerosité auxquelles la criminologie a été trop peu attentive.

Un autre article critique intéressant est celui de José Rico sur les législations hispano-américaines. La conclusion de l'auteur est dure mais logique, compte tenu de sa démonstration. Il y est indiqué que les lois hispano-américaines dites de dangerosité sociale représentent un bon exemple de dispositions qui ne pourront jamais 
atteindre l'objectif de justice sociale, visant à la création d'une société meilleure. Elles s'attaquent à des situations qui constituent davantage des problèmes sociaux non résolus que des questions proprement pénales. Quant à la finalité théorique originale de prévention, elle est un échec car elle a été détournée vers des considérations politiques, économiques, moralisantes et répressives, au grand détriment du principe de légalité et de son corollaire, la protection des droits des individus. L'argument favorable au maintien de ce type de dispositions, consistant à invoquer le nombre restreint de cas où ces lois s'appliquent, ne devrait pas être pris en considération. L'histoire montre comment, lorsque des situations problématiques surgissent, le pouvoir les a presque toujours utilisées telles quelles. Tant que les législations de dangerosité sociale seront là, elles constitueront une très forte tentation pour certains gouvernements et représenteront pour les citoyens des grands risques de violation de leurs droits fondamentaux.

\section{CONCLUSION}

Le dernier mot du livre est écrit par Manuel Lopez-Rey qui en profite pour élargir la critique de la notion de dangerosité en une critique de la discipline criminologique. Il signale, à juste titre, que la combinaison "dangerosité-déviance-dissidence "est fréquemment appliquée pour masquer sous le couvert de thèses scientifiques une persécution politique et ce, tant dans les pays capitalistes que marxistes. La criminologie, selon l'auteur, est une discipline extrêmement conservatrice. La criminologie est incapable de résoudre le problème actuel de la justice pénale. Il propose de réduire radicalement l'importance attachée à la personnalité du délinquant comme pivot criminologique. Selon Lopez-Rey, la personnalité doit être prise en considération mais en rapport avec d'autres postulats parmi lesquels celui de la justice sociale est plus important. Une telle justice requiert une conception de la justice de nature socio-politique qui, dans son application, doit s'efforcer autant que possible de réduire certaines inégalités, et assurer, d'autre part, le respect des droits humains à titre individuels et collectifs.

Tout compte fait, ce volume sur la "dangerosité et justice pénale » est un excellent outil de réflexion critique autant pour le praticien que pour l'universitaire.

S'il est toujours vrai que "du choc des idées " devraient nâ̂tre des pistes d'actions prometteuses pour " rendre vraiment justice », ce dossier y contribuera à sa façon ! 


\section{BIBLIOGRAPHIE}

DEBUYST, C. (1975) : "Les nouveaux courants dans la criminologie contemporaine: la mise en cause de la psychologie criminelle et de son objet a, Revue de droit pénal et de criminologie, 845-870.

DEBUYST, C. (1977) : "Le concept de dangerosité et un de ses éléments constitutifs : la personnalité (criminelle) "), Déviance et socićté, 363-387.

DEBUYST, C. (1978): «L'étiologie envisagée dans le cadre des études cliniques : de la notion de personnalité criminelle à celle du comportement délinquant ", Congrès international de criminologie, Lisbonne.

DEBUYST, C. sous la dir. de (1981): Dangerosité et justice pénale : ambigüté d'une pratique, Genève, Médecine et Hygiène, Paris, Masson, Collection Déviance et société. 350 pages.

DEBUYST, C. (1982) : La personnalité criminelle, Genève, Médecine et Hygiène, Paris, Masson, Collection Déviance et société, livre en préparation.

KINBERG, OLOF (1959): Les problèmes fondamentaux de la criminologie, Centre Irançais de droit comparé, Paris, Cujas, 325 pages.

MANNHEIM. HERMANN (1953): "Etude sociologique du délinquant adulte ", Revue internationale de politique criminelle, $\mathrm{n}^{0} 3,63-68$.

MONTANDON, C. (1979) : "Actualités bibliographiques : la dangerosité, revue de littérature anglo-saxonne ". Déviance et société, 89-104.

PINATEL, JEAN (1970) : Criminologie, Tome III, in Bouzat, Pierre et Pinatel, Jean, Traité de droit pénal et de criminologie. $2^{\mathrm{e}}$ éd., Paris, Dalloz, 660 pages.

SHAH. S.A., J. DOZOIS, M. LALONDE, J. POUPART ET M. FOUCAULT (1981): "Débat : le dompteur face à la dangerosité ", Déviance et société, 369-422.

VAN BEMMELEN, J. (1953) : "L'étude sociologique de cas individuel घ, fer Cours International de Criminologie. Paris, 15 sept. - 24 oct. 1952; Melun, Imprimerie administrative, 502-510. 